
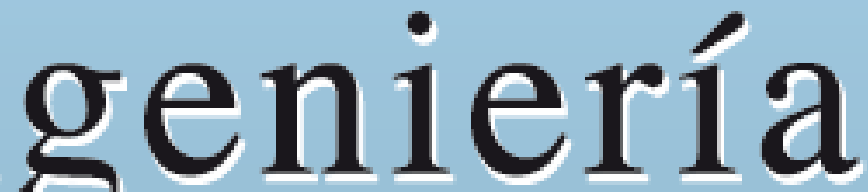

Revista de la Universidad de Costa Rica ENEROIDICIEMBRE 2002 - VOLUMEN 12 - $\mathrm{N}^{\circ} 1$ y 2

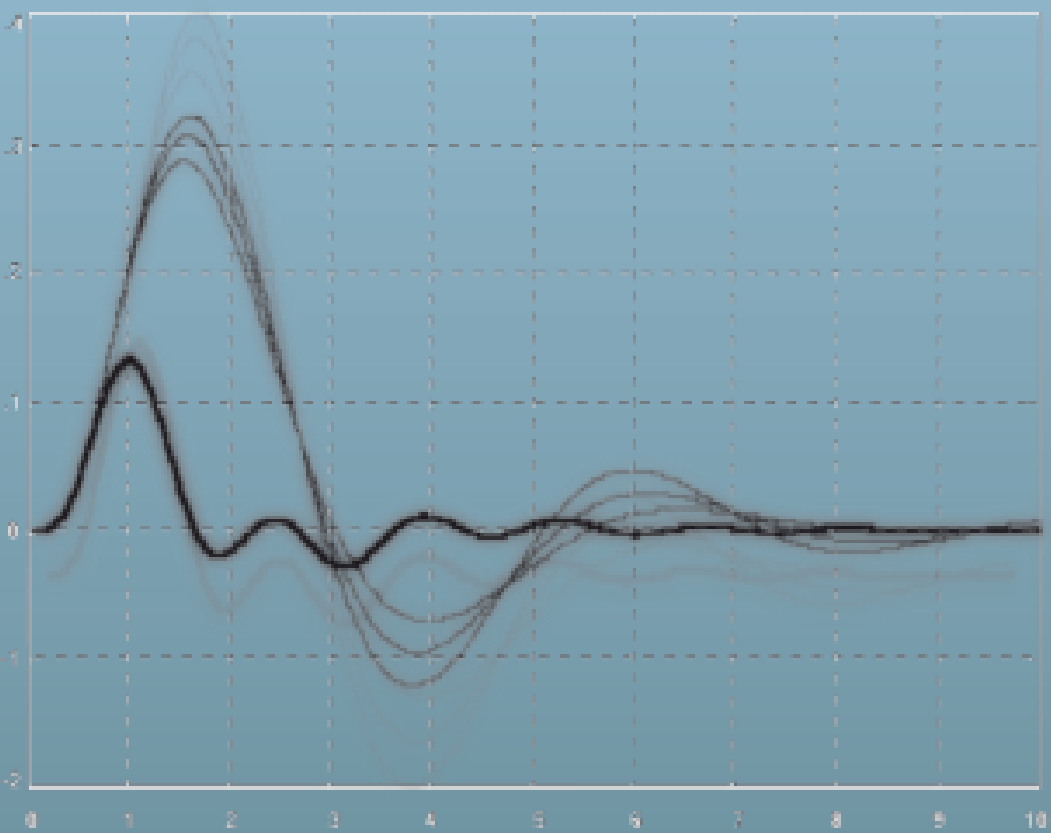




\title{
APLICACIÓN DEL C.A.E. EN INGENIERÍA ESTRUCTURAL
}

\section{Eduardo Lima Calvo}

\begin{abstract}
Resumen
En este artículo se define el CAE (Computer Aided Engineering) o ingeniería asistida por computadora, y se exploran sus diferencias con el CAD y CAM. Se hace un recorrido desde los primeros intentos de las aplicaciones del CAE, hasta el momento en que tanto las metodologías y algoritmos de cálculo, como el software y hardware disponibles se compaginaron para ofrecer programas de cómputo verdaderamente amigables y funcionales. De entre estos programas se elige y revisa una marca específica de gran difusión en el medio ingenieril costarricense, y se finaliza con un ejemplo de aplicación de estas tecnologías de punta, en la resolución de un análisis no lineal estático de una edificación pequeña.
\end{abstract}

Palabras clave: Ingeniería de software realizada por computador, ingeniería de sistemas, ingeniería de estructuras.

\begin{abstract}
In this article is defined the CAE (Computer Aided Engineering), and their differences with the CAD and CAM are explored. A journey is made from the first intents of the applications of the CAE, until the moment in that so much the methodologies and calculation algorithms, as the software and available hardware were paged to offer truly friendly and functional computation programs. Of among these programs it is chosen and it revises a specific mark of great diffusion in Costa Rica. It concludes with an example of application of these tip technologies in the resolution of a static non lineal analysis of a small construction.
\end{abstract}

\section{INTRODUCCIÓN}

La ingeniería civil es una profesión tan antigua como el ser humano. Recordemos la prehistoria, cuando las primeras tribus humanas comenzaron a dejar la protección de las cavernas para diseminarse por los territorios, convirtiéndose así en asentamientos de agricultores. El hombre sintió la necesidad de construir albergues que los protegieran primero de las inclemencias del clima y de los depredadores, y posteriormente de otras tribus expansionistas. Con el pasar de los miles de años, hasta nuestros días es que dentro de la ingeniería civil se pudieron diferenciar algunas ramas de especialidad, como lo es la ingeniería estructural, que en un país como el nuestro se conoce más bien como la ingeniería sismorresistente.

Se debe hacer especial acotación al origen mismo de la palabra ingeniería, el cual proviene de la palabra ingenio, por lo tanto se podría decir que la ingeniería es el ingenio mismo aplicado. Siendo la informática y la computación una ciencia producida por el hombre mismo, esta ha incursionado todos los campos de acción proveyendo de soluciones o simplemente "modos más sencillos de hacer las cosas". En este último punto la ingeniería estructural no es ajena al gran desarrollo que ha permitido no sólo el uso intensivo de programas de cómputo, sino también algo tan sencillo como las calculadoras que vinieron a sustituir a las famosas "reglas de cálculo".

Son innumerables las anécdotas que cualquier ingeniero puede relatar sobre sus primeras experiencias con el mundo de la computación, cuando cursaba sus primeros cursos universitarios, y la comparación con la actualidad.

\section{ALCANCE}

Este estudio no pretende ser un tratado riguroso de historia de las aplicaciones computacionales en el campo de la ingeniería estructural, sino más bien un repaso por hechos relevantes de 
conocimiento común en el medio profesional costarricense, así como un vistazo a las fronteras actuales del análisis estructural asistido por computadora.

\section{DEFINICIÓN DE CAE}

El CAE es un término que se utiliza para referirse a la Computer-Aided Engineering, Ingeniería Asistida por Computadora, es decir a la utilización de computadoras para analizar y diseñar elementos (Tech Target, 2002). Se diferencia del CAD (Computer-Aided Design) en que este último ha venido a referirse especialmente al proceso de dibujo, diseño y modelado gráfico, más que a un proceso de aplicaciones matemáticas para el cálculo de elementos (Tech Target, 2002). Otro término diferente es el CAM (Computer-Aided Manufacturing), que está más relacionado con los procesos de robótica o fabricación industrial automatizada que con procesos de cálculo (Tech Target, 2002).

Siendo el CAE un término tan amplio, a un nivel más cercano se refiere entonces a los paquetes de software que brindan una plataforma para el análisis y el diseño en las ingenierías mecánica y civil en todas sus especialidades. Es por esto último que recientemente han pasado a denominarse mas bien CASE (Computer-Aided Software Engineering).

\section{PRIMERAS APLICACIONES}

El verdadero desarrollo de los CAE, para pequeñas y medianas empresas de ingeniería (PYMES), se inicia alrededor de los años 1981 a 1983 cuando el lenguaje de programación FORTRAN se vuelve confiable, luego de 3 años de su lanzamiento inicial, y los estudiantes universitarios tienen un acceso, aunque limitado aún, a una computadora central que pueda manejar la información introducida en ella por medio de tarjetas perforadas.
Al revolucionarse el mundo académico con la aparición de las primeras computadoras personales (PC), se da igualmente una revolución en el desarrollo y uso de programas de cómputo de auxilio a los tediosos cálculos de sistemas estructurales complejos, cambiando totalmente la orientación del análisis estructural y encaminándolo hacia sistemas de resoluciones matriciales fácilmente operables por las computadoras, ya no sólo por las más grandes, sino también por pequeñas computadoras. Es en esos primeros años que un ingeniero estructural, profesor de la Universidad de California en Berkeley, el Dr. Edward L. Wilson, desarrolla en lenguaje de programación FORTRAN-77 una serie de subrutinas de programación conocidas como el Computer Adaptive Language (CAL), las cuales sirven de plataforma para adaptar el programa SOLIDSAP desarrollado por él mismo en 1970 (Computers \& Structures, 2002), y usado extensivamente en proyectos de gran magnitud e importancia, y crear así una serie de programas llamados Structural Analysis Programs (SAP). El primero de la serie SAP capacitado para operar en pequeñas computadoras fue denominado SAP-80, dado que su utilización masiva sería en esa década, y el programa de última generación de la serie SAP, conocido como SAP-2000, se ha convertido en uno de los software de uso más intensivo en nuestro medio, gracias en parte a la formación recibida en el estado de California de E.E.U.U., de algunos de los profesores de ingeniería estructural de la Universidad de Costa Rica.

\section{USO DE LA TÉCNICA DEL ELEMENTO FINITO}

La técnica de disgregar una estructura en sus componentes individuales e indivisibles conocidos como análisis de elementos finitos (FEA), es la base del modelado de las estructuras, y de la incorporación de la información correspondiente, en forma tal que pueda ser introducida al computador para su análisis e interpretación (Structural Research \& Analysis Corp, 2002). 
Desde sus primeras versiones, el SAP ha contado con una apropiada librería de elementos finitos para modelar: elementos longitudinales, planos y elementos de cascarón bidimensionales, así como sólidos tridimensionales. También esta técnica permite modelar superficies de soporte infinitas al usar un conglomerado de elementos finitos en un grado de subdivisión tal que permite alcanzar la exactitud deseada, con la única limitante de la capacidad y velocidad del equipo de cómputo, que sin embargo a los niveles actuales, en realidad no representa limitación.

\section{INGRESO DE DATOS INTERACTIVOS EN TIEMPO REAL}

Si nos remontamos a la época cuando salió a la venta la serie de programas SAP-80 podemos entender porqué se tuvo tanto éxito. Anteriormente, para la entrada de datos al programa se debía utilizar el llamado formato rígi$d o$, heredado del antiguo ingreso de datos en tarjetas perforadas. La novedad vino con el formato libre de ingreso de información, que permitía utilizar un procesador de palabras comercial para guardar los datos en lenguaje ASCII sin tener que seguir las reglas de espaciamiento rígido y permitiendo incorporar identificadores para los datos, como por ejemplo: A para el área de los elementos, $\mathbf{F}$ para las fuerzas, etc., y seguidamente escribir el dato numérico a continuación de un signo igual (=) que se colocaba tras el identificador. Sin embargo, esta entrada de datos seguía siendo muy fría, susceptible de muchas equivocaciones del digitador, y requería una gran inversión de tiempo en la preparación de la información que sería introducida, y debía ser corroborada examinando cuidadosamente la "salida" de un pre-procesador que graficaba la estructura antes de la "corrida" que calculaba y analizaba la estructura. La interpretación de los datos sí se realizaba en una forma más interactiva, ya que los módulos de pos-proceso generaban un modelo tridimensional que graficaba los diagramas de fuerzas internas y la estructura deformada.

Con la serie de programas SAP-90 se dió el primer intento de alcanzar una entrada de datos interactiva con la creación del módulo de preproceso llamado SAPIN. Sin embargo aún se tenían muchos problemas. El ingreso de datos interactivo en tiempo real se alcanza finalmente con la serie SAP-2000 que integra todos los módulos de preproceso, cálculo y pos-proceso, además de los de diseño de acero y concreto, para alcanzar un producto altamente eficiente del punto de vista de tiempo de generación e interpretación de la información por parte del usuario.

\section{FAMILIA DE PROGRAMAS DE COMPUTERS \& STRUCTURES, INC}

La compañía Computers \& Structures, Inc es la propietaria y distribuidora de los programas de análisis y diseño estructural de la serie SAP, y actualmente su cartera de productos incluye 3 series: SAP, ETABS y SAFE dedicados al análisis de obras civiles en general, de edificios de varios pisos, y de losas y cimentaciones de concreto armado, respectivamente.

A continuación se presenta una cronología de la aparición de cada una de las series y sus transformaciones.

A. Serie SAP

$\begin{array}{lr}\text { Structural Analysis } & \text { Program } \\ \text { SAP } & 1970 \\ \text { SOLIDSAP } & 1971 \\ \text { SAP3 } & 1972 \\ \text { SAPIV } & 1973 \\ \text { SAP80 } & 1979 \\ \text { SAP90/SAPIN } & 1989 \\ \text { SAP2000 } & 1998\end{array}$

B. Serie ETABS

Extended Three Dimensional Analysis of Building Systems

$\begin{array}{ll}\text { TABS } & 1972 \\ \text { TABS1 } 1 & 1973 \\ \text { TABS84 } & 1984 \\ \text { ETABS } & 1975 \\ \text { ETABS / AISC } & 1979 \\ \text { SUPER-ETABS } & 1980 \\ \text { ETABS84 } & 1984 \\ \text { ETABS5 } & 1990\end{array}$




$\begin{array}{ll}\text { ETABS6/ ETABSIN } & 1996 \\ \text { ETABS7 } & 2000 \\ \text { ETABS8 } & 2002\end{array}$

C. SERIE SAFE

Slab Analysis based on Finite Element

$\begin{array}{ll}\text { SAFE } & 1978 \\ \text { SAFE } & 2000\end{array}$

\section{ANÁLISIS NO LINEAL - SAP2000}

Desde que se comprendió el impacto que los programas diseñados para usarse en computadoras personales podrían tener en el análisis estructural, se pensó no sólo en el análisis lineal estático o dinámico, sino que se pensó en la potencialidad del análisis no lineal estático, o Static Push Over Analysis. Esto último se vió reforzado por las nuevas normativas norteamericanas en materia de estudios de vulnerabilidad sísmica de edificios existentes y su posterior reforzamiento estructural (Habibullah and Pyle, 1998), y también en nuestro país (Acuña, 1998). Avanzando hasta los límites, se puede pensar aún más en el análisis no lineal dinámico o Incremental Dynamic Analysis (Vamvatsikos and Cornell, 2002). Si por otro lado, nos posicionamos desde la óptica de la filosofía de diseño sismorresistente basado en
Sistemas de Protección, o Control del Comportamiento de las Estructuras (Vertero, 1998), entenderemos la facilidad que nos brindan los métodos numéricos programados para incorporar en el modelo elementos de aislamiento en la base (Fernández y Gutiérrez, 1998), así como elementos pasivos o activos de disipación de energía por desplazamiento o velocidad.

La última generación de la serie de programas SAP es una herramienta sumamente poderosa para el análisis estructural. Este programa permite realizar un análisis no lineal (Static Push Over), ya que tiene la capacidad de modelar las rótulas plásticas debidas a la fluencia en elementos sometidos a flexión, carga axial, flexocompresión y fuerza cortante. Otra característica importante es la posibilidad de incorporar elementos de vínculo de tipo no lineal en la estructura, como por ejemplo: aisladores de base por deslizamiento o péndulo, amortiguadores viscoelásticos, y separadores en tensión y compresión.

Para ilustrar mejor la completa capacidad de esta nueva Ingeniería Asistida por Computadora (CAE) se ha desarrollado un ejemplo sencillo a partir de un edificio ficticio de concreto armado de 3 niveles ( 2 entrepisos). Ver Figura .1.

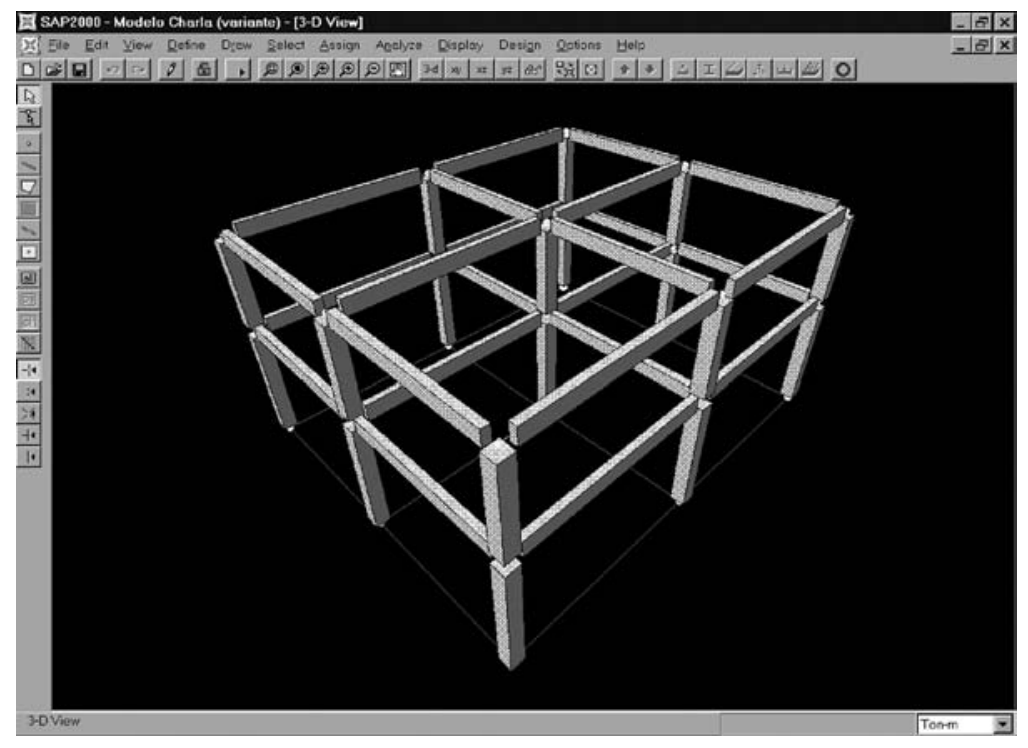

Figura 1. Ejemplo ilustrativo Fuente: Impresión SAP2000. 


\section{A. Descripción}

Edificio de concreto armado de 3 niveles, 2 entrepisos. El sistema estructural resistente son marcos de concreto dúctiles conformados por columnas de $0.50 \times 0.50 \mathrm{~m}$, vigas de carga de $0.20 \times 0.50 \mathrm{~m}$ y vigas de amarre de $0.20 \times 0.45$ $\mathrm{m}$. El entrepiso es de concreto presforzado de viguetas de $0.15 \mathrm{~m}$ y una losa de concreto de 0.05 $\mathrm{m}$ de espesor. Las paredes internas estarán desligadas de la estructuras principal y serán de bloques de concreto de $0.12 \mathrm{~m}$ de espesor. El techo será de láminas de hierro galvanizado y cerchas metálicas. El uso del inmueble será para oficinas.

\section{B. Parámetros sísmicos}

Por importancia:

Grupo B

Por forma estructural:

Tipo 1

Vida útil:

Probabil. excedencia: 50 años

Período retorno:

$40 \%$

100 años

Perfil /estrato:

Ductilidad:

Amortiguamiento:

Suelo

firme

6

Aceleración máxima:

0,05

$18 \%(\mathrm{~g})$

\section{Masas}

W1:

W2:

\section{Periodos naturales}

T1 (desplazam. X):

T2 (desplazam. Y):

$0,40 \mathrm{~s}$

T3 (rotación Z):

$0,19 \mathrm{~s}$

El programa se encarga del análisis dinámico para resolver la ecuación de valores propios y encontrar los modos fundamentales de vibración de la estructura.

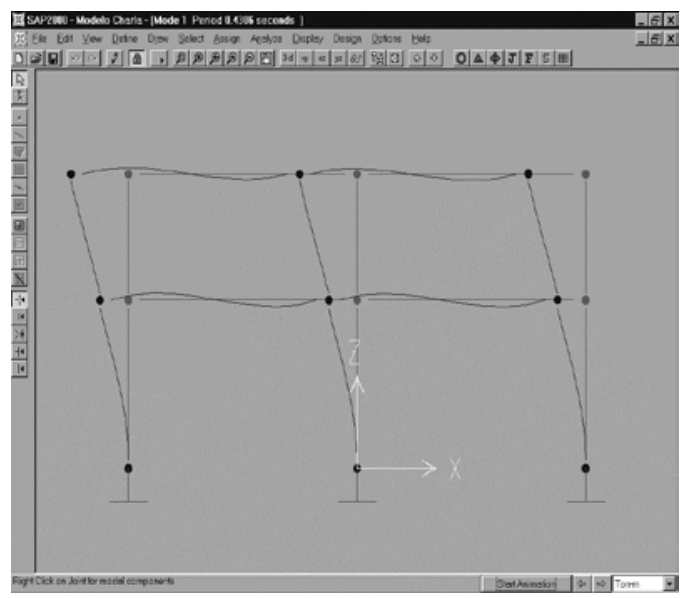

Figura 2. Modo de vibración 1 (Elevación).

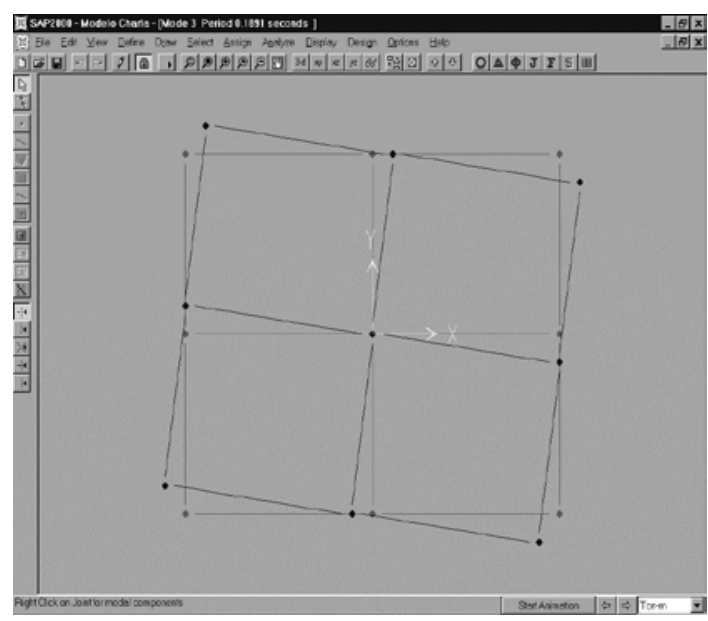

Figura 3. Modo de vibración 3 (Planta). 
E. Cortantes y desplazamientos por piso (Sismo en $\mathrm{X}$ )

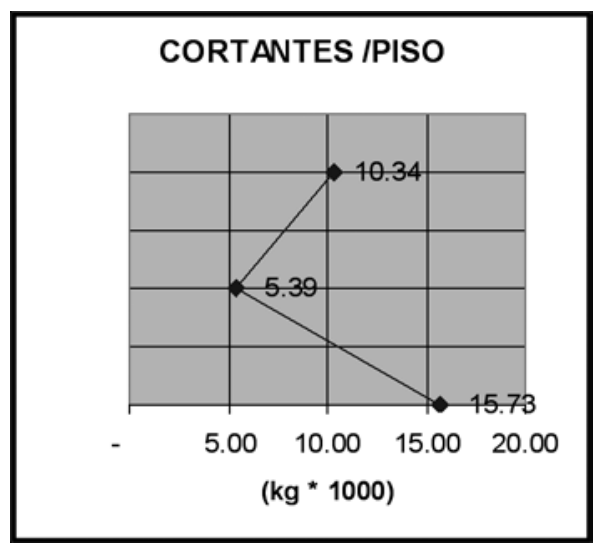

Figura 4. Cortantes por Piso.

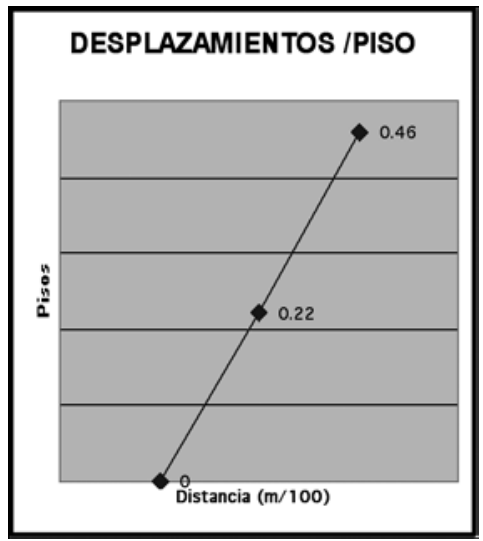

Figura 5. Desplazamientos/Piso.

F. Diseño de elementos.

El programa incluye módulos de diseño para concreto armado y acero, según distintos códigos de diseño. En el caso del ejemplo, se especificó el uso del código de diseño ACI-89.

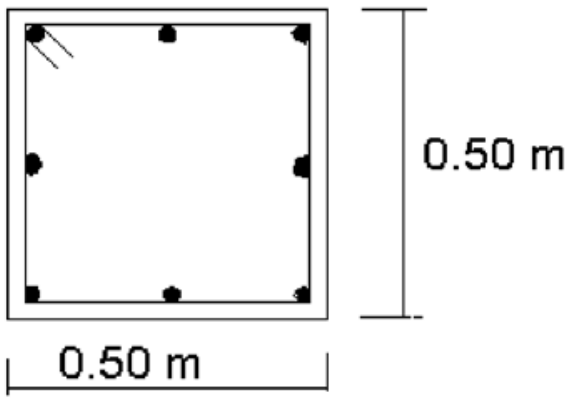

8 var. \#7

Figura 6. Columna típica.

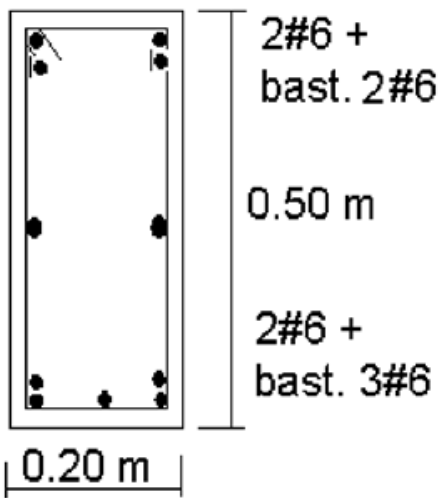

Figura 7. Viga Carga.

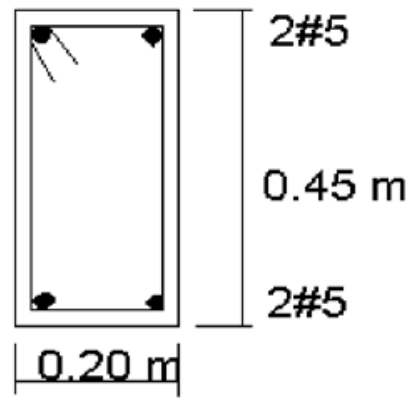

Figura 8. Viga amarre. 


\section{G. ANÁLISIS NO LINEAL}

Una vez que ya se tienen diseñados los elementos de la estructura, vigas y columnas, es posible determinar las magnitudes de las fuerzas internas que producen el estado último en los elementos, y por ende la aparición de rótulas plásticas por deformaciones de rotación debidas a la flexión. Para ello el programa incluye el modelado de rótulas plásticas, las cuales se asignan a los elementos en las zonas de aparición esperada de las mismas, de acuerdo a las propiedades autocalculadas por el programa, o a información suministrada por el usuario.

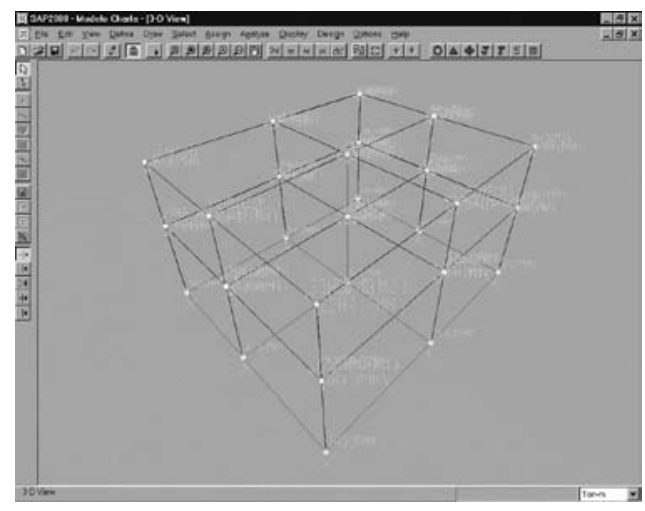

Figura 9. Asignación de las propiedades de rótulas.

Se acumulan las diferentes pérdidas de redundancia hiperestática, hasta que se alcance la deformación límite fijada para el análisis, o la pérdida de estabilidad de la estructura.

Finalmente es posible graficar toda la información y obtener diagramas de esfuerzo-deformación para la estructura en el rango no lineal.

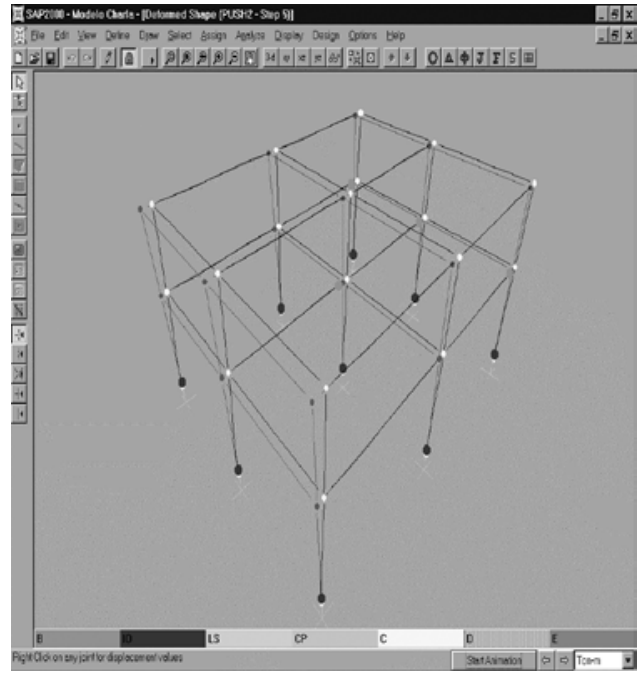

Figura 10. Secuencia de formación de rótulas plásticas.

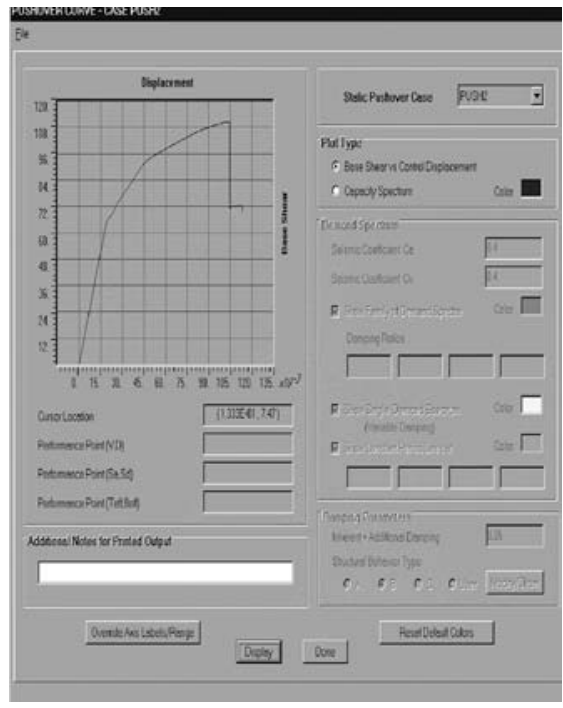

Figura 11. Diagrama Cortante en la Base vrs. Desplazamiento. 


\section{BIBLIOGRAFÍA}

\section{Bibliografía general}

1. Acuña-Prado, R.; Propuesta para un Nuevo Capítulo del Código Sísmico de Costa Rica sobre Estudios de Vulne-rabilidad y Adecuación de Estructuras Existentes. Memorias del X Seminario Latinoamericano de Ingeniería Sísmica. San José: 1998.

2. Arrieta-Torrealba, José María, et. al.; Un programa de elementos finitos: SAP-90. Madrid: Centro de Estudios y Experimentación de Obras Públicas, 1992.

3. Bertero, V;. Uso de Sistemas de Protección en el Diseño Sismorresistente. Memorias del X Seminario Latinoamericano de Ingeniería Sísmica. San José: 1998.

4. Fernández, C. et. al.; Comportamiento de Edificios de Varios Pisos con Aislamiento Sísmico en su Base. Memorias del X Seminario Latinoamericano de Ingeniería Sísmica. San José: 1998.

5. Habibullah, Ashraf; ETABS. Three Dimensional Analysis of Building Systems. U.S.A.: Computers \& Structures Inc., 1990.

6. Habibullah, Ashraf and Pyle, Stephen; "Practical Three Dimensional Nonlinear Static Pushover Analysis”. Structure Magazine. (Winter, 1998).

7. Wilson, Edward L.; Hollings, J. P. y Dovey, H. H.; ETABS. Three Dimensional Analysis of Building Systems (Extended Version). U.S.A.: University of California, 1979.
8. Wilson, Edward L. y Habibullah, Ashraf; SAP80. Structural Analysis Programs. U.S.A.: Computers \& Structures Inc., 1984.

9. Wilson, Edward L; Structural Analysis on Microcomputers.

\section{Bibliografía digital}

1. Computers and Structures, Inc. "Information Company."(http://www.csiberkeley.com/Company.htm). February 2002.

2. Structural Research \& Analysis Corp. "What is Finite Element Analysis (FEA)?." (http://www.cosmosm.com/support/fea1.htm). February 2002.

3. Tech Target. "CAE." (http://whatis.techtarget.com/definition/0,,sid9_gci758962,00. html). February 2002.

4. Vamvatsikos, Dimitrios and Cornell, Allin."The Incremental Dynamic Analysis and Its Applicationto Performance-Based EarthquakeEngineering."(http://blume. stanford.edu/pdffiles/Vamvatsikos_Dimitr ios.pdf). February 2002.

\section{SOBRE EL AUTOR}

\section{Ing. Eduardo Lima Calvo}

Consultor Independiente

Tel: 394-0081 Fax: 262-3682

Correo Electrónico: elima@cfia.or.cr 